\title{
EVOLUTION EQUATIONS HAVING CONSERVATION LAWS WITH FLUX CHARACTERISTICS
}

\author{
B. VAN BRUNT ${ }^{\varpi 1}$ and M. VLIEG-HULSTMAN ${ }^{1}$
}

(Received 9 August 2006)

\begin{abstract}
A class of evolution equations in divergence form is studied in this paper. Specifically, we develop conditions under which the spatial divergence term, the flux, corresponds to the characteristic of a conservation law. The $\mathrm{KdV}$ equation is a prominent example of an equation having a flux term that is also a characteristic for a conservation law. We show that the flux term must be self-adjoint. General equations for the corresponding conservation laws and Hamiltonian densities are derived and supplemented with examples.
\end{abstract}

2000 Mathematics subject classification: primary 35K.

Keywords and phrases: evolution equations, conservation laws, Hamiltonian densities.

\section{Introduction}

In this paper we study conservation laws for evolution equations of the form

$$
u_{t}+Q_{x}=0,
$$

where $Q$ is a smooth function of $\mathbf{u}=\left(u, u_{1}, u_{2}, \ldots, u_{n}\right)$. Here, $u_{k}$ denotes the $k$ th partial derivative of $u$ with respect to $x$, and $u_{0}=u$. In particular, we focus on a class of evolution equations (1.1) that have the property that the flux term $Q$ is also the characteristic for a conservation law. A prominent example of such an equation is the $\mathrm{KdV}$ equation

$$
u_{t}+\left(u_{2}+3 u^{2}\right)_{x}=0
$$

Recall that if a partial differential equation $\mathscr{F}\left(\mathbf{u}, u_{t}, u_{n+1}\right)=0$ has a conservation law with characteristic $Q(\mathbf{u})$, then there exists a function $\mathbf{P}(\mathbf{u})=\left(P_{1}(\mathbf{u}), P_{2}(\mathbf{u})\right)$ such

\footnotetext{
${ }^{1}$ Institute of Fundamental Sciences, Mathematics, Massey University, New Zealand; email:B.vanBrunt@massey.ac.nz

(C) Australian Mathematical Society 2007, Serial-fee code 1446-1811/07
} 
that.

$$
Q(\mathbf{u}) \mathscr{F}\left(\mathbf{u}, u_{t}, u_{n+1}\right)=\nabla \cdot \mathbf{P}(\mathbf{u}),
$$

where $\nabla$ denotes $(\partial / \partial t, \partial / \partial x)=\left(D_{t}, D_{x}\right)$. Note that Equation (1.3) must be satisfied for all $\mathbf{u}$. If $\mathbf{u}$ is such that $\mathscr{F}\left(\mathbf{u}, u_{t}, u_{n+1}\right)=0$, then $\nabla \cdot \mathbf{P}(\mathbf{u})=0$ represents a conservation law.

Conservation laws are often derived via Noether's theorem, which requires a Lagrangian formulation. Following Anco and Bluman [1,2], and van Brunt et al. [8], however, this formulation can be bypassed by recognizing that the kernel of the Euler operator is the space of total divergences. The particulars of this method are discussed in the above references and in Olver [5].

The class of differential equations (1.1) involves operators that depend on $u_{t}$ and $\mathbf{u}$, so that the corresponding Euler operator for a function $K\left(\mathbf{u}, u_{t}, u_{n+1}\right)$ is

$$
E(K)=K_{u}-D_{x} K_{u_{1}}-D_{t} K_{u_{1}}+D_{x}^{2} K_{u_{2}}-D_{x}^{3} K_{u_{3}}+\cdots+(-1)^{n+1} D_{x}^{n+1} K_{u_{n+1}} .
$$

Here we use the notation $D_{x}^{k+1}=D_{x} D_{x}^{k}$ for $k=0,1, \ldots$, and $K_{u_{k}}=\partial K / \partial u_{k}$ denotes the partial derivative of $K$ holding $u$ and $u_{j}$ fixed for $j \neq k$. Suppose that $Q(\mathbf{u})$ is a characteristic for a conservation law of $\mathscr{F}\left(\mathbf{u}, u_{t}, u_{n+1}\right)=0$. Then, there is a $\mathbf{P}$ such that Equation (1.3) is satisfied and hence

$$
E(Q \mathscr{F})=E(\nabla \cdot \mathbf{P})=0 .
$$

Equation (1.4) is particularly simple for operators of the form $\mathscr{F}=u_{t}+D_{x} Q$. Since, for any smooth functions $A$ and $B, E(A+B)=E(A)+E(B)$, Equation (1.4) implies

$$
E\left(Q\left(u_{t}+D_{x} Q\right)\right)=E\left(Q u_{t}\right)+E\left(Q D_{x} Q\right)=0
$$

however,

$$
Q D_{x} Q=\frac{1}{2} D_{x} Q^{2}=\nabla \cdot\left(0, \frac{Q^{2}}{2}\right),
$$

and hence Equation (1.4) reduces to

$$
E\left(Q u_{t}\right)=0 \text {. }
$$

Equation (1.5) is thus a condition on the flux $Q$ for the partial differential equation (1.1) to have a conservation law with $Q$ as a characteristic. Equations of the form (1.1) that satisfy (1.5) will be called flux characteristic.

Any first-order equation of the form (1.1) is flux characteristic. In this case

$$
Q=Q(u), \quad \text { and hence } \quad E\left(Q u_{t}\right)=E(\nabla \cdot(R(u), 0))=0,
$$

where $R_{u}=Q$. A conservation law corresponding to this characteristic is thus

$$
\nabla \cdot\left(R, Q^{2} / 2\right)=0 \text {. }
$$

In contrast, no even-order equation of the form (1.1) can be flux characteristic. This result is given in Corollary 2.2 . 


\section{General Results for Higher-Order Equations}

In this section we derive results for equations of the form (1.1), where $n>1$. The third-order case $(n=2)$ is discussed in more detail in the next section. Suppose that $Q=Q\left(u, u_{1}, \ldots, u_{n}\right)$, then Equation (1.5) is

$$
E\left(Q u_{t}\right)=Q_{u} u_{t}-D_{x}\left(u_{t} Q_{u_{1}}\right)-D_{t}(Q)+D_{x}^{2}\left(u_{t} Q_{u_{2}}\right)+\cdots+(-1)^{n} D_{x}^{n}\left(u_{t} Q_{u_{n}}\right)=0 .
$$

For notational simplicity, let

$$
u_{t k}=\frac{\partial^{k+1} u}{\partial t \partial x^{k}}
$$

with $u_{t 0}=u_{t}$. Now,

$$
D_{x}^{m}\left(u_{t} Q_{u_{j}}\right)=\sum_{k=0}^{m}\left(\begin{array}{l}
m \\
k
\end{array}\right) u_{t k} D_{x}^{m-k}\left(Q_{u_{j}}\right),
$$

so that Equation (2.1) expands to the form

$$
\begin{aligned}
E\left(Q u_{t}\right)= & u_{t} Q_{u}-\sum_{k=0}^{n} u_{t k} Q_{u_{k}}-\sum_{k=0}^{1}\left(\begin{array}{l}
1 \\
k
\end{array}\right) u_{t k} D_{x}^{1-k}\left(Q_{u_{1}}\right)+\sum_{k=0}^{2}\left(\begin{array}{l}
2 \\
k
\end{array}\right) u_{t k} D_{x}^{2-k}\left(Q_{u_{2}}\right) \\
& +\cdots+(-1)^{n} \sum_{k=0}^{n}\left(\begin{array}{l}
n \\
k
\end{array}\right) u_{t k} D_{x}^{n-k}\left(Q_{u_{n}}\right)=0
\end{aligned}
$$

Collecting coefficients of $u_{t k}$ gives

$$
E\left(Q u_{t}\right)=\sum_{j=0}^{n} u_{t j} W_{j}
$$

where

$$
W_{j}=-Q_{u_{j}}+\sum_{k=0}^{n-j}(-1)^{j+k}\left(\begin{array}{c}
j+k \\
j
\end{array}\right) D_{x}^{k}\left(Q_{u_{j+k}}\right) .
$$

If $Q$ is a characteristic for a conservation law of Equation (1.1), then Equation (1.5) is satisfied for all $u$; hence the coefficients of $u_{i k}$ must vanish for $k=0, \ldots, n$. In summary, we have the following result.

THEOREM 2.1. Equation (1.1) is flux characteristic if and only if for all $u$ we have that $Q$ satisfies

$$
W_{j}=0, \quad j=0, \ldots, n
$$

where $W_{j}$ is defined by Equation (2.2). 
COROLLARY 2.2. Equation (1.1) is flux characteristic only if $n$ is even. Hence, only equations of odd order can be flux characteristic.

PROOF. This result follows immediately from (2.3). Suppose that $n$ is odd and that $Q_{u_{n}} \neq 0$. Putting $j=n$ in (2.3) gives

$$
W_{n}=\left(-1+(-1)^{n}\right) Q_{u_{n}}=0,
$$

which implies $Q_{u_{n}}=0$. We thus conclude that $n$ must be even and hence partial differential equations of the form (1.1) that are flux characteristic must be of odd order.

COROLLARY 2.3. Suppose that Equation (1.1) is flux characteristic and $n>2$. Then $Q$ must be of the form

$$
Q=\alpha u_{n}+\beta u_{n-1}^{2}+\gamma u_{n-1}+\delta,
$$

where $\alpha=\alpha\left(u, u_{1}, \ldots, u_{n-2}\right), \delta=\delta\left(u, u_{1}, \ldots, u_{n-2}\right)$,

$$
\begin{aligned}
& \beta=\frac{n}{4} \alpha_{u_{n-2}} \text { and } \\
& \gamma=\frac{n}{2}\left(D_{x} \alpha-\alpha_{u_{n-2}} u_{n-1}\right) .
\end{aligned}
$$

PROOF. The form of $Q$ follows from (2.3) with $j=n-1$, noting that $n$ must be even. Specifically, the relation

$$
W_{n-1}=-2 Q_{u_{n-1}}+n D_{x} Q_{u_{n}}=0
$$

must hold for all $u$ and hence the partial derivatives of $W_{n-1}$ with respect to the $u_{j}$, $j=0, \ldots, n+1$, must vanish. Expanding the total derivative, the above equation is

$$
2 Q_{u_{n-1}}=n \sum_{k=0}^{n} Q_{u_{n} u_{k}} u_{k+1}
$$

Now, differentiating $W_{n-1}$ with respect to $u_{n+1}$ gives

$$
Q_{u_{n} u_{n}}=0,
$$

and differentiating $W_{n-1}$ with respect to $u_{n}$ gives

$$
-2 Q_{u_{n-1} u_{n}}+n\left(\sum_{k=0}^{n} Q_{u_{n} u_{n} u_{k}} u_{k+1}+Q_{u_{n-1} u_{n}}\right)=0,
$$


and Equation (2.7) thus implies

$$
(n-2) Q_{u_{n-1} u_{n}}=0 .
$$

Since $n>2$, the above expression gives

$$
Q_{u_{n-1} u_{n}}=0 \text {. }
$$

Differentiating $W_{n-1}$ with respect to $u_{n-1}$ and using Equations (2.7) and (2.8) we have

$$
2 Q_{u_{n-1} u_{n-1}}=n Q_{u_{n-2} u_{n}}
$$

and differentiating both sides of the above expression with respect to $u_{n-1}$ yields

$$
2 Q_{u_{n-1} u_{n-1} u_{n-1}}=n Q_{u_{n-2} u_{n-1} u_{n}}=0
$$

where Equation (2.8) has been used. We thus have

$$
Q_{u_{n-1} u_{n-1} u_{n-1}}=0 \text {. }
$$

Equations (2.7), (2.8) and (2.10) imply that $Q$ must be of the form

$$
Q=\alpha u_{n}+\beta u_{n-1}^{2}+\gamma u_{n-1}+\delta
$$

where $\alpha, \beta, \gamma$ and $\delta$ are functions of $u, u_{1}, \ldots, u_{n-2}$. Using the above expression for $Q$, Equation (2.9) implies Equation (2.4) and Equation (2.5) comes directly from Equation (2.6).

Recall that the Fréchet derivative of $Q=Q\left(u, u_{1}, \ldots, u_{n}\right)$ acting on a function $F=F\left(u, u_{1}, \ldots, u_{n}\right)$ is

$$
D_{Q}(F)=\sum_{k=0}^{n} Q_{u_{k}} D_{x}^{k} F,
$$

and the adjoint is

$$
D_{Q}^{\#}(F)=\sum_{k=0}^{n}(-1)^{k} D_{x}^{k}\left(Q_{u_{k}} F\right)
$$

The Euler operator can be expressed in terms of the adjoints of Fréchet derivatives, for example,

$$
E(Q F)=D_{Q}^{\#}(F)+D_{F}^{\#}(Q)
$$

A Fréchet derivative $D_{Q}$ is self-adjoint if $D_{Q}(F)=D_{Q}^{\#}(F)$ for any smooth function $F=F\left(u, u_{1}, \ldots, u_{n}\right)$. The next theorem shows that flux characteristic equations are characterized by self-adjoint Fréchet derivatives.

THEOREM 2.4. Equation (1.1) is flux characteristic if and only if

$$
D_{Q}=D_{Q}^{\#}
$$


PROOF. For any smooth function $F=F\left(u, u_{1}, \ldots, u_{n}\right)$,

$$
D_{Q}^{\#}(F)=\sum_{j=0}^{n}\left(\sum_{k=0}^{n-j}(-1)^{j+k}\left(\begin{array}{c}
j+k \\
j
\end{array}\right) D_{x}^{k}\left(Q_{u_{j+k}}\right)\right) D_{x}^{j}(F) ;
$$

hence

$$
D_{Q}^{\#}(F)-D_{Q}(F)=\sum_{j=0}^{n} W_{j} D_{x}^{j}(F),
$$

where $W_{j}$ is defined by Equation (2.2). If $D_{Q}$ is self-adjoint, then the above expression must vanish for all $F$; hence $W_{j}=0$ for all $j=0, \ldots, n$. Theorem 2.1 thus implies that Equation (1.1) is flux characteristic. If Equation (1.1) is flux characteristic then Theorem 2.1 implies that $W_{j}=0$ for all $j=0, \ldots, n$ and hence $D_{Q}$ is selfadjoint.

We note here that self-adjoint conservation law characteristics are generic to evolution equations. In other words, if $Q$ is a characteristic of a conservation law for an equation of the form

$$
u_{t}+G\left(u, u_{1}, \ldots, u_{n}\right)=0,
$$

then $D_{Q}$ must be self-adjoint. The expression

$$
E\left(Q\left(u_{t}+G\right)\right)=E\left(Q u_{t}\right)+E(Q G)=0,
$$

must hold for all $u$, and $t$ derivatives occur only in the term $E\left(Q u_{t}\right)$. Every term of $E\left(Q u_{t}\right)$ has a $t$ derivative, and this leads to

$$
E\left(Q u_{t}\right)=0 \text {, }
$$

which in turn produces (2.3).

Equation (1.1) has a Hamiltonian density if there exists a function $\mathscr{H}$ such that

$$
E(\mathscr{H})=Q \text {. }
$$

Equation (2.11) does not uniquely define $\mathscr{H}$ because

$$
E\left(\mathscr{H}+D_{x} F\right)=E(\mathscr{H})=Q
$$

for any smooth function $F$.

COROLLARY 2.5. Equation (1.1) is flux characteristic if and only if there exists a function $\mathscr{H}$ such that $E(\mathscr{H})=Q$. The function $\mathscr{H}$ can be constructed using the homotopy formula

$$
\mathscr{H}=\int_{0}^{1} u Q(\lambda \mathbf{u}) d \lambda,
$$

where $Q(\lambda \mathbf{u})=Q\left(\lambda u, \lambda u_{1}, \ldots, \lambda u_{n}\right)$. 
PROOF. The corollary follows immediately from Theorem 2.4 and the Helmholz condition (see, for example, Olver, op. cit., page 355). Specifically, Theorem 2.4 shows that (1.1) is flux characteristic if and only if $D_{Q}$ is self-adjoint. The Helmholz condition asserts that there exists a function $\mathscr{H}$ such that $E(\mathscr{H})=Q$ if and only if $D_{Q}$ is self-adjoint. The reader is directed to Olver loc. cit. for a proof of the homotopy formula.

The above corollary shows that flux characteristic equations are characterized by the existence of a Hamiltonian density. The equation

$$
u_{t}+(E(\mathscr{H}))_{x}=0
$$

must have a conservation law with characteristic $E(\mathscr{H})$ for any smooth function $\mathscr{H}=\mathscr{H}\left(u, u_{1}, \ldots, u_{m}\right)$.

Third and higher-order equations that are flux characteristic have at least three distinct conservation law characteristics.

COROLLARY 2.6. Suppose that Equation (1.1) is fiux characteristic. Then $Q_{0}=1$, $Q_{1}=u$ and $Q_{3}=Q$ are characteristics for conservation laws.

ProOF. Since Equation (1.1) is in divergence form, it is clear that $Q_{0}=1$ is a characteristic for a conservation law, and $Q_{3}=Q$ must be a characteristic of a conservation law from the definition of a flux characteristic equation. The only result to establish is that $Q_{1}=u$ is also a characteristic, and this follows immediately from the self-adjointness of $Q$. Specifically, it is evident that $u$ is self-adjoint and that $E\left(u u_{t}\right)=0$. Now,

$$
\begin{aligned}
E\left(u D_{x} Q\right) & =E\left(D_{x}(u Q)-u_{1} Q\right)=-E\left(u_{1} Q\right)=-\left(D_{Q}^{\#}\left(u_{1}\right)+D_{u_{1}}^{\#}(Q)\right) \\
& =-\left(D_{Q}\left(u_{1}\right)+D_{u_{1}}^{\#}(Q)\right)=-\left(D_{x} Q-D_{x} Q\right)=0
\end{aligned}
$$

hence $Q_{1}=u$ is also a characteristic for a conservation law.

\section{Third-Order Equations}

In this section we focus on conservation laws for equations of the form (1.1), where

$$
Q=Q\left(u, u_{1}, u_{2}\right)
$$

Note that Corollary 2.3 does not include this case; however, it is straightforward to glean the general form of $Q$ from (2.3). If Equation (1.1) is flux characteristic, 
then (2.3) must be satisfied for $j=0,1,2$. The proof of Corollary 2.2 shows that $W_{2}=0$. The remaining equations are

$$
\begin{aligned}
& W_{1}=Q_{u_{1}}-D_{x}\left(Q_{u_{2}}\right)=0 \text { and } \\
& W_{0}=D_{x}\left(Q_{u_{1}}-D_{x}\left(Q_{u_{2}}\right)\right)=0 .
\end{aligned}
$$

Evidently, $W_{0}=0$ if $W_{1}=0$ so that Equation (3.1) is the only independent equation. Expanded, Equation (3.1) is

$$
Q_{u_{1}}-Q_{u_{2}} u_{1}-Q_{u_{1} u_{2}} u_{2}-Q_{u_{2} u_{2}} u_{3}=0 .
$$

Now, $Q$ does not depend on $u_{3}$, and Equation (3.2) must be satisfied for all $u$. We thus conclude that the coefficient of $u_{3}$ must vanish and hence $Q$ is of the form

$$
Q\left(u, u_{1}, u_{2}\right)=A\left(u, u_{1}\right) u_{2}+B\left(u, u_{1}\right) .
$$

Substituting the above expression for $Q$ into Equation (3.2) yields the condition

$$
B_{u_{1}}=A_{u} u_{1} \text {. }
$$

Any smooth function $Q$ of the form (3.3) that satisfies (3.4) also satisfies Equation (1.5) and hence the corresponding equation is flux characteristic. Equation (3.4) is evidently the condition for $D_{Q}$ to be self-adjoint. Note that if $Q$ satisfies Equation (3.4), then there is a function $\Phi$ such that

$$
u_{1} Q=D_{x} \Phi,
$$

where $\Phi_{u_{1}}=A u_{1}$ and $\Phi_{u}=B$. It follows from Equation (3.4) that

$$
\Phi=\int A u_{1} d u_{1} .
$$

Corollary 2.5 shows that if the equation is flux characteristic, then there is a function $\mathscr{H}$ such that $E(\mathscr{H})=Q$. Equation (2.12) provides a homotopy formula for $\mathscr{H}$. For the third-order case, however, (2.3) is tractable and a Hamiltonian density can be constructed directly. Once $\mathscr{H}$ is determined, a conservation law corresponding to the characteristic $Q$ can be constructed explicitly without reference to the homotopy formula for a conservation law (see, for example, Anco and Bluman [1,2] for the homotopy formula).

If Equation (2.11) is satisfied for all $u$, then

$$
-D_{x} \mathscr{H}_{u_{1}}+\mathscr{H}_{u}=-\mathscr{H}_{u_{1} u_{1}} u_{2}-\mathscr{H}_{u u_{1}} u_{1}+\mathscr{H}_{u}=A u_{2}+B
$$

so that any function $\mathscr{H}\left(u, u_{1}\right)$ that satisfies the equations

$$
A=-\mathscr{H}_{u_{1} u_{1}} \quad \text { and } \quad B=\mathscr{H}_{u}-u_{1} \mathscr{H}_{u_{u_{1}}}
$$


is a Hamiltonian density for the third-order case of Equation (1.1). A solution to these equations is given by

$$
\mathscr{H}=\int B d u-u_{1} \int A d u_{1}+K\left(u_{1}\right)+D_{x} F(u),
$$

where $F$ is an arbitrary smooth function and $K_{u_{1}}=u_{1} A-\int B_{u_{1}} d u$. Now,

$$
Q u_{t}=E(H) u_{t}=\left(\mathscr{H}_{u}-D_{x} \mathscr{H}_{u_{1}}\right) u_{t}=\mathscr{H}_{u} u_{t}+\mathscr{H}_{u_{1}} u_{t 1}-D_{x}\left(u_{t} \mathscr{H}_{u_{1}}\right)=\nabla \cdot \mathbf{R} \text {, }
$$

where $\mathbf{R}=\left(\mathscr{H},-u_{t} \mathscr{H}_{u_{1}}\right)$. A conservation law corresponding to $Q$ is thus

$$
\nabla \cdot\left(\mathscr{H},-u_{1} \mathscr{H}_{u_{1}}+\frac{(E(\mathscr{H}))^{2}}{2}\right)=0 .
$$

Corollary 2.6 also shows that $u$ is a characteristic for a conservation law. The conservation law corresponding to this characteristic is

$$
\nabla \cdot\left(\frac{u^{2}}{2}, u Q-\Phi\right)
$$

where $\Phi$ is given by Equation (3.5). The equation is in divergence form and hence

$$
\nabla \cdot(u, Q)
$$

is also a conservation law. In summary, we have the following result.

THEOREM 3.1. A third-order equation of the form (1.1) is flux characteristic if and only if $Q$ is of the form (3.3), where $A$ and $B$ satisfy Equation (3.4). In this case a conservation law corresponding to $Q$ is given by Equation (3.7), where $\mathscr{H}$ is defined by Equation (3.6). Conservation laws are also given by Equations (3.8) and (3.9).

EXAMPLE 1. The KdV equation (1.2) has a flux of the form

$$
Q=u_{2}+3 u^{2} \text {. }
$$

Evidently Equation (3.4) is satisfied and hence the equation is flux characteristic. Equation (3.6) gives a Hamiltonian density

$$
\mathscr{H}=-\frac{u_{1}^{2}}{2}+u^{3}
$$

The homotopy formula (2.12) gives

$$
\begin{aligned}
\mathscr{H} & =\int_{0}^{1} u Q(\lambda \mathbf{u}) d \lambda=u \int_{0}^{1}\left(\lambda u_{2}+3 \lambda^{2} u^{2}\right) d \lambda=\frac{u u_{2}}{2}+u^{3} \\
& =\frac{1}{2} D_{x}\left(u u_{1}\right)-\frac{u_{1}^{2}}{2}+u^{3},
\end{aligned}
$$


so that the densities are equivalent up to a total derivative. Equation (3.7) yields the conservation law

$$
\nabla \cdot\left(-\frac{u_{1}^{2}}{2}+u^{3}, u_{t} u_{1}+\frac{\left(u_{2}+3 u^{2}\right)^{2}}{2}\right)=0 .
$$

We note that the $u_{t}$ can be eliminated at this stage using the evolution equation itself, that is, $u_{t}=-D_{x}\left(u_{2}+3 u^{2}\right)$.

A close relation to the KdV equation is the KdVB equation

$$
u_{t}+\left(\mu u_{2}-v u_{1}+u^{2}\right)_{x}=0,
$$

where $\mu$ and $\nu$ are constants. Here, $A=\mu$ and $B=-v u_{1}+u^{2}$, so that Equation (3.4) is satisfied only if $\nu=0$.

EXAMPLE 2. The equation studied by Dey $[3,4]$ in divergence form is

$$
u_{t}+\left(c u_{2}+a \frac{u^{n+1}}{n+1}+a b \frac{u^{2 n+1}}{2 n+1}\right)_{x}=0,
$$

where $a, b$ and $c$ are constants, $c \neq 0$ and $n$ is a positive integer. Here, $Q=A u_{2}+B$, where $A=c$, and

$$
B=a \frac{u^{n+1}}{n+1}+a b \frac{u^{2 n+1}}{2 n+1} .
$$

Equation (3.4) is satisfied and hence the equation is flux characteristic. A Hamiltonian density is

$$
\mathscr{H}=-\frac{c u_{1}^{2}}{2}+a \frac{u^{n+2}}{(n+1)(n+2)}+a b \frac{u^{2 n+1}}{(2 n+1)(2 n+2)},
$$

and the corresponding conservation law is

$$
\nabla \cdot\left(\mathscr{H}, c u_{t} u_{1}+\frac{Q^{2}}{2}\right)=0
$$

EXAMPLE 3. Petersson et al. [6] considered a third-order equation of the form

$$
u_{t}+u^{\tau} u_{3}+n u_{1} u_{2}+m u_{1}^{3}+r u_{2}+p u_{1}^{2}+q u_{1}+s=0,
$$

where $n, m, r, p, q$ and $s$ are functions of $u$, and $\tau$ is a real constant. For the cases $\tau=0$ and $\tau=3$, they studied the functional forms of $n, m, r, p, q$ and $s$ such that the equation is integrable in the sense that it has an infinite number of Lie-Bäcklund 
symmetries. The above equation can be put into divergence form only if $s=$ constant, $r^{\prime}(u)=p(u)$ and

$$
n^{\prime}(u)-2 m(u)=\tau(\tau-1) u^{\tau-2} .
$$

If, in addition $s=0$, the equation can be written in the form (1.1), where

$$
Q=u^{\tau} u_{2}+\left(n-\tau u^{\tau-1}\right) \frac{u_{1}^{2}}{2}+r u_{1}+f
$$

and $f^{\prime}(u)=q(u)$. Equation (3.4) shows that the p.d.e. is flux characteristic if $r=0$ and $n(u)=2 \tau u^{\tau-1}$. The class of equations that are flux characteristic is thus

$$
u_{t}+u^{\tau} u_{3}+2 \tau u^{\tau-1} u_{1} u_{2}+\frac{\tau(\tau-1)}{2} u^{\tau-2} u_{1}^{3}+q u_{1}=0 .
$$

The Hamiltonian density is

$$
\mathscr{H}=f(u)-\frac{u^{\mathrm{r}} u_{i}^{2}}{2}
$$

and the conservation law corresponding to the characteristic $Q$ is

$$
\nabla \cdot\left(f(u)-\frac{u^{\tau} u_{1}^{2}}{2}, u^{\tau} u_{1} u_{1}+\frac{Q^{2}}{2}\right) .
$$

\section{Fifth-Order Equations}

We now look at fifth-order equations of the form (1.1) that are flux characteristic. Corollary 2.3 shows that $Q$ must be of the form

$$
Q=\alpha u_{4}+\beta u_{3}^{2}+\gamma u_{3}+\delta
$$

where $\alpha, \beta, \gamma$ and $\delta$ are functions of $u, u_{1}$ and $u_{2}$, and $\beta$ and $\gamma$ are given by Equations (2.4) and (2.5), that is,

$$
\beta=\alpha_{u_{2}} \quad \text { and } \quad \gamma=2\left(\alpha_{u} u_{1}+\alpha_{u_{1}} u_{2}\right)
$$

Corollary 2.5 implies that $Q$ has a Hamiltonian density $\mathscr{H}$. Once a Hamiltonian density is determined, the corresponding conservation law is

$$
\nabla \cdot\left(H, D_{x}\left(u, \mathscr{H}_{u_{2}}\right)-2\left(u_{11} \mathscr{H}_{u_{2}}\right)-u_{1} \mathscr{H}_{u_{1}}+\frac{Q^{2}}{2}\right)=0
$$

Equations such as the fifth-order KdV equation

$$
u_{t}+\left(u_{4}+10 u u_{2}+5 u_{1}^{2}+10 u^{3}\right)_{x}=0
$$


the Kuperschmidt equation

$$
u_{t}-\left(u_{4}+\frac{5}{2} u_{2} u+\frac{15}{8} u_{1}^{2}+\frac{5}{12} u^{3}\right)_{x}=0
$$

and the Caudrey-Dodd-Gibbon-Sawada-Kotera equation

$$
u_{t}+\left(u_{4}+30 u u_{2}+60 u^{3}\right)_{x}=0 \text {, }
$$

are of the form

$$
u_{t}+\left(\alpha u_{4}+F\left(u, u_{1}, u_{2}\right)\right)_{x}=0,
$$

where $\alpha$ is a constant. The test for self-adjointness and the calculation of the Hamiltonian density are particularly tractable for this form. Briefly, if $Q=R+S$ is self-adjoint and $R$ is self adjoint, then

$$
E\left((R+S) u_{t}\right)=E\left(R u_{t}\right)+E\left(S u_{t}\right)=E\left(S u_{t}\right)=0 ;
$$

hence $S$ must also be self-adjoint. Now, $R=u_{4}$ is self-adjoint; hence, if Equation (4.5) is flux characteristic, $F$ must be self-adjoint. In essence, if Equation (4.5) is flux characteristic then it must be of the form

$$
u_{t}+\left(\alpha u_{4}+A u_{2}+B\right)_{x}=0,
$$

where $A$ and $B$ satisfy Equation (3.4). Evidently, if $\mathscr{K}$ is a Hamiltonian density for the equation

$$
u_{t}+\left(A u_{2}+B\right)_{x}=0
$$

then a Hamiltonian density for Equation (4.6) is

$$
\mathscr{H}=\alpha \frac{u_{2}^{2}}{2}+\mathscr{K} .
$$

EXAMPLE 4. For the KdV equation (4.2), $A=10 u$ and $B=5 u_{1}^{2}+10 u^{3}$. Equation (3.4) is satisfied and hence the fifth-order $\mathrm{KdV}$ equation is flux characteristic. A Hamiltonian density is given by

$$
\mathscr{H}=\frac{u_{2}^{2}}{2}-5 u_{1}^{2} u+\frac{5}{2} u^{4}
$$

Equation (4.1) gives the conservation law

$$
\nabla \cdot(\mathscr{H}, X)=0
$$

where, for

$$
\begin{aligned}
& Q=u_{4}+10 u u_{2}+5 u_{1}^{2}+10 u^{3}, \\
& X=u_{t} u_{3}-u_{t 1} u_{2}+10 u u_{t} u_{1}+\frac{Q^{2}}{2} .
\end{aligned}
$$


For the Kuperschmidt equation (4.3), $A=5 u / 2$ and $B=15 u_{1}^{2} / 8+5 u^{3} / 12$. Here, Equation (3.4) is not satisfied and hence the Kuperschmidt equation is not flux characteristic. It is straightforward to see that Equation (4.4) is also not flux characteristic.

EXAMPLE 5. Tan et al. [7] studied the general fifth-order $\mathrm{KdV}$ equation

$$
u_{t}+u_{5}+u_{3}+(N(\mathbf{u}))_{x}=0 \text {, }
$$

where, for constants $\alpha_{k}, k=0,1,2,3$,

$$
N(\mathbf{u})=\alpha_{0} u^{2}+\alpha_{1} u u_{2}+\alpha_{2} u_{1}^{2}+\alpha_{3} u^{3} .
$$

The above equation can be written as

$$
u_{t}+\left(u_{4}+A u_{2}+B\right)_{x}=0
$$

where $A=\alpha_{1} u+1$ and $B=\alpha_{0} u^{2}+\alpha_{2} u_{1}^{2}+\alpha_{3} u^{3}$. The equation is flux characteristic if $A$ and $B$ satisfy Equation (3.4), and this leads to the condition $\alpha_{1}=2 \alpha_{2}$ in agreement with Tan.. A Hamiltonian density is given by

$$
\mathscr{H}=\frac{u_{2}^{2}}{2}+\alpha_{0} \frac{u^{3}}{3}-\alpha_{2} u u_{1}^{2}+\alpha_{3} \frac{u^{4}}{4}-\frac{u_{1}^{2}}{2} .
$$

A conservation law corresponding to the characteristic $Q=u_{4}+A u_{2}+B$ is

$$
\nabla \cdot(\mathscr{H}, X)=0,
$$

where

$$
X=D_{x}\left(u_{t} u_{2}\right)-2\left(u_{t 1} u_{2}\right)+u_{t}\left(2 \alpha_{2} u u_{1}+u_{1}\right)+\frac{Q^{2}}{2}
$$

\section{References}

[1] S. C. Anco and G. W. Bluman, "Derivation of conservation laws from nonlocal symmetries of differential equations", J. Math. Phys. 37 (1996) 2361-2375.

[2] S. C. Anco and G. W. Bluman, "Direct construction of conservation laws from field equations", Phys. Rev. Lett. 78 (1997) 2869-2873.

[3] B. Dey, "Domain wall solutions of KdV like equations with higher order nonlinearity", J. Phys. A: Math. Gen 19 (1986) L9-L12.

[4] B. Dey, "KdV-like equations with domain wall solutions and their Hamiltonians", in Solitons: Introduction and Applications: Proceedings Winter School on Solitons, Tiruchirapalli, India, Jan. 1987 (ed. M. Lakshmanan), (Springer-Verlag, New York, 1988), 367-374.

[5] P. J. Olver, Applications of Lie Groups to Differential Equations (Springer-Verlag, New York, 1986). 
[6] N. Petersson, N. Euler and M. Euler, "Recursion operators for a class of integrable third-order evolution equations", Stud. Appl. Math. 112 (2004) 201-225.

[7] Y. Tan, J. Yang and D. Pelinovsky, "Semi-stability of embedded solitons in the general fifth-order KdV equation", Wave Motion 36 (2002) 241-255.

[8] B. van Brunt, D. Pidgeon, M. Vlieg-Hulstman and W. Halford, "Conservation laws for second-order parabolic partial differential equations", ANZIAM J. 45 (2004) 333-348. 